\title{
Spinal Interneurons Facilitate Coactivation of Hand Muscles during a Precision Grip Task in Monkeys
}

\author{
Tomohiko Takei ${ }^{1,2}$ and Kazuhiko Seki ${ }^{1,2,3,4}$ \\ ${ }^{1}$ Department of Neurophysiology, National Institute of Neuroscience, Tokyo 187-8502, Japan, ${ }^{2}$ Department of Developmental Physiology, National Institute \\ for Physiological Sciences and ${ }^{3}$ School of Life Science, The Graduate University for Advanced Studies, Okazaki 444-8585, Japan, and ${ }^{4}$ PRESTO, Japan \\ Science and Technology Agency, Kawaguchi, Saitama 332-0012, Japan
}

Grasping is a highly complex movement requiring coordination of a number of hand joints and muscles. In contrast to cortical descending systems, the contribution of the subcortical system for coordinating this higher degree of freedom is largely unknown. Here we explore how spinal interneurons (INs) contribute to the coordination of hand muscles by recording their activity from the cervical spinal cord (C5-T1) simultaneously with electromyographic (EMG) activity from hand and arm muscles in three monkeys performing a precision grip task. Spike-triggered averages of the rectified EMGs were compiled for 255 neurons (4821 neuron-muscle pairs). Twentysix neurons produced 68 significant postspike effects in hand and arm muscles and were identified as premotor interneurons (PreM-INs), which presumably have relatively direct synaptic effects on spinal motoneurons. The majority of the PreM-INs (22/26 neurons) produced postspike effects in finger muscles (intrinsic and extrinsic hand muscles) compared with wrist (9/26 neurons) and elbow muscles (1/26 neurons). The effects in finger muscles were mostly facilitative [postspike facilitations (PSFs), 19/22 neurons], and few had suppressive effects (postspike suppressions, 3/22 neurons). Moreover, PreM-INs produced more divergent PSFs in intrinsic hand muscles (2.5 \pm 1.9 muscles/neuron) than in wrist muscles (1.2 \pm 0.4 muscles/neurons). We conclude that spinal PreM-INs produce divergent facilitations preferentially in intrinsic hand muscles. These results suggest that spinal interneurons contribute to the control of hand grasping in primates by combining and coordinating multiple finger muscles.

\section{Introduction}

Grasping is a highly developed hand movement in human and other primates. Elucidating the neural mechanism for the control of grasping is still challenging after the pioneering work by Sir Charles Bell (Bell, 1834), primarily because of the anatomical complexity of the primate hand, which consists of 27 muscles in humans. How the CNS coordinates this high degree of freedom is one of the central questions of motor control. Among the various types of grasping (Napier, 1956), the precision grip is an important evolutionary advance in primates and is thought to be crucially controlled through the corticospinal tract (CST), as damage to the CST by stroke or spinal cord injury causes severe impairment of precision grip (Lawrence and Kuypers, 1968; Porter and Lemon, 1993; Lemon, 2008).

It is known that the CST controls the upper limb motoneurons via direct and indirect pathways (Rathelot and Strick, 2009). A direct corticomotoneuronal (CM) connection from the primary motor cortex to the spinal motoneurons has been a primary

Received Aug. 15, 2010; revised Sept. 22, 2010; accepted Oct. 20, 2010.

This work was supported by Grants-in-Aid for Scientific Research on Priority Areas ("Mobilligence" and "System Study on Higher-Order Brain Function") from Ministry of Education, Culture, Sports, Science, and Technology (MEXT) (18020030, 18047027), a Grant-in-Aid for Young Scientists (B) from MEXT (21700437), and Japan Science and Technology Agency PRESTO program. We thank Nobuaki Takahashi and Kaoru Isa (National Institute for Physiological Sciences) for technical assistance.

Correspondence should be addressed to Dr. Kazuhiko Seki, Department of Neurophysiology, National Institute of Neuroscience, 4-1-1 Ogawa-Higashi, Kodaira, Tokyo 187-8502, Japan. E-mail: seki@ncnp.go.jp.

DOI:10.1523/JNEUROSCI.4297-10.2010

Copyright $\odot 2010$ the authors $\quad 0270-6474 / 10 / 3017041-10 \$ 15.00 / 0$ subject in investigations of CST control of precision grip and fine finger movements (Muir and Lemon, 1983; Buys et al., 1986; Lemon et al., 1986; Bennett and Lemon, 1996; Schieber and Rivlis, 2005; Lemon, 2008). For example, some CM cells are principally active during precision grip (Muir and Lemon, 1983), and they selectively facilitate small groups of finger muscles (Buys et al., 1986; Bennett and Lemon, 1996). Thus, these direct connections are crucial in the control of fractionated finger movement.

In contrast, little is known about the function of indirect CM connections for the control of precision grip. Anatomical studies have demonstrated that the spinal target of descending projections from the cortical and brainstem motor areas is mainly the spinal intermediate zone rather than the ventral horn and motoneuron pools (Kuypers and Brinkman, 1970; Kuypers, 1981; Cheema et al., 1984; Ralston and Ralston, 1985; Robinson et al., 1987; Bortoff and Strick, 1993; Dum and Strick, 1996; Armand et al., 1997). Last-order interneurons (INs), which project to motoneurons monosynaptically, are mainly localized in this intermediate zone (Jankowska, 1992), and they may integrate peripheral afferent information with the descending motor command (Baldissera et al., 1981; Jankowska, 1992). Therefore, it is reasonable to assume that the precision grip is also controlled through these spinal INs. Although the spinal IN activity of monkeys performing wrist movements has been reported already (Maier et al., 1998; Perlmutter et al., 1998), no previous studies have examined spinal IN activity during primate grasping. As a result, it is unknown whether spinal IN-mediated pathways contribute to the control of precision grip. 
Here we explore the contribution of spinal INs in the control of grasping by recording their activity in the cervical cord of awake monkeys performing a precision grip task and identifying their influence on the hand and finger muscles with spike-triggered averages of electromyographic (EMG) activity. We found a significant number of spinal premotor interneurons (PreM-INs) that projected to finger motoneurons. These PreM-INs often produced divergent facilitations in multiple finger muscles rather than affecting a single muscle, indicating that spinal INs could produce coactivation of finger muscles characteristic of grasping movements. These results suggest that the neural pathway mediated by spinal PreM-INs is involved in the divergent control of hand synergies, which may be a platform of stability required for the superimposition of precision control.

Preliminary results of this study have been presented in abstract form (Takei and Seki, 2009).

\section{Materials and Methods}

Recordings were made from three adult male macaque monkeys (monkey A: Macaca fuscata, $6.8 \mathrm{~kg}$; monkey E: Macaca mulatta, $5.6 \mathrm{~kg}$ and monkey U: $M$. fuscata, $8.5 \mathrm{~kg}$ ). The experiments were performed in accordance with the National Institutes of Health Guidelines for the Care and Use of Laboratory Animals and were approved by the Animal Research Committee at the National Institute for Physiological Sciences, Okazaki, Japan.

Behavioral task. Monkeys were trained to sit in a monkey chair with their left elbow restrained while they performed a precision grip task (Fig. $1 A)$. The monkey inserted a thumb and index finger through separate holes in a horizontal plate to access the manipulandum levers (Takei and Seki, 2008). The manipulandum comprised two spring-loaded levers, each with a potentiometer (model 357, Vishay Spectrol) fitted to its pivot point to report lever position and a touch sensor $(30-100 \mathrm{pF}$; D5C1DA0, Omron) to measure finger contact with the levers. The binary state of both touch sensors was constantly monitored throughout the experiments to determine when thumb and index finger were both in contact with the levers. A trial would not begin until both touch sensors were activated and would abort immediately upon loss of contact with either lever. A strain gauge was attached to each lever for measuring the force exerted by both fingers. Thumb and index finger positions were continuously presented to the monkey via the positions of two rectangle cursors displayed on a screen in front of the monkey (Fig. 1A,B). Two target boxes were also displayed, and the monkey was required to keep each cursor inside its target box during a trial. Each trial began with the presentation of two target boxes positioned to make the thumb and index finger $3.0 \mathrm{~cm}$ apart (Fig. $1 \mathrm{~B}$, "Rest"). After 1.0-2.0 s, the "out" targets disappeared and two "center" targets appeared simultaneously (Fig. $1 B$, "Go"), signaling the monkey to flex its thumb and index finger to bring the cursors into the center targets (Fig. $1 \mathrm{~B}$, "Grip"). The required displacement of both fingers by $\sim 1.0 \mathrm{~cm}$, which corresponded to $\sim 1.0 \mathrm{~N}$ force. The monkey was required to maintain the lever positions within the center targets for 1.0-2.0 s (Fig. $1 B$, "Hold") then release them back to the "out" targets (Fig. $1 B$, "Release") once the "center" target disappeared (Fig. 1B, "Go"). Successful completion of a trial was rewarded with a drop of applesauce (Fig. $1 B$, "Reward"). On average, $\sim 1500$ successful trials/recording session with the success rate of $\sim 80 \%$ were performed.
B

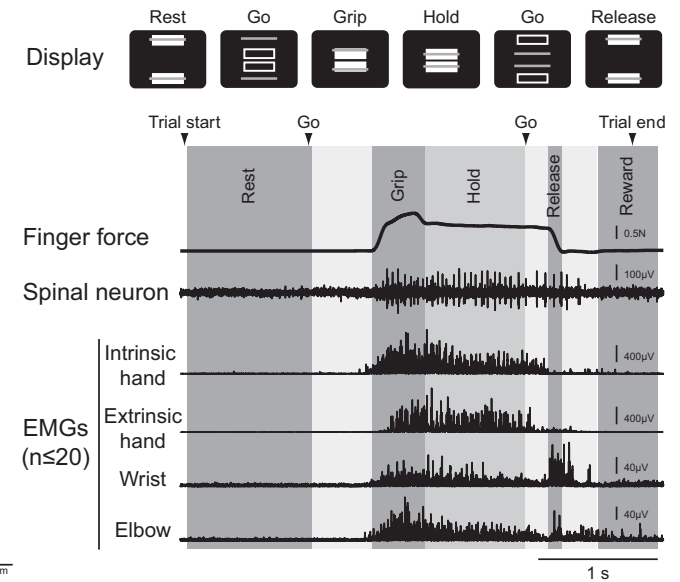

$1 \mathrm{~s}$

Figure 1. Experimental setup and recording sites. A, Recording configuration. Monkeys sat in front of a computer screen and

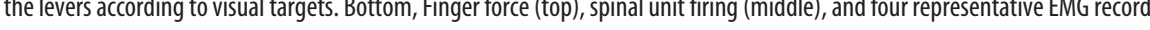

Surgical operations. After behavioral training was complete, three separate surgeries were performed to implant a head restraint, a recording chamber over the cervical spinal cord, and EMG wire electrodes into multiple forelimb muscles. All surgeries were performed on different days using isoflurane $\left(1.0-2.0 \%\right.$ in $\left.2: 1 \mathrm{O}_{2}: \mathrm{N}_{2} \mathrm{O}\right)$ or sevoflurane anesthesia $\left(1.5-3.0 \%\right.$ in $\left.2: 1 \mathrm{O}_{2}: \mathrm{N}_{2} \mathrm{O}\right)$ and under aseptic conditions. During the head restraint and spinal recording chamber surgeries, the monkey was immobilized with intravenous pancuronium bromide $(0.05 \mathrm{mg} / \mathrm{kg}$ every hour; Mioblock, Organon) and artificially respired. Respiration rate was adjusted to keep end-tidal $\mathrm{CO}_{2}$ within $20-25 \mathrm{mmHg}$. Two head restraints (plastic tubes) were fixed to the skull with titanium screws and dental acrylic. An oval-shaped spinal recording chamber (Perlmutter et al., 1998) was implanted over the lower cervical spinal cord. C3-T2 vertebrae were exposed bilaterally, and titanium screws were inserted into the lateral mass of each vertebra. After performing a unilateral laminectomy of the $\mathrm{C} 4-\mathrm{C} 7$ vertebrae, the recording chamber was positioned over the laminectomy and cemented in place with dental acrylic. For recording EMGs, pairs of stainless steel wires (AS632, Cooner Wire) were implanted subcutaneously in 20 forelimb muscles of monkeys A and E, including intrinsic hand muscles [first, second, third and forth dorsal interosseous (FDI, 2DI, 3DI, and 4DI, respectively), adductor pollicis (ADP), abductor pollicis brevis (AbPB), abductor digiti minimi $(\mathrm{AbDM})$ ], extrinsic hand muscles [flexor digitorum superficialis (FDS), radial and ulnar parts of flexor digitorum profundus (FDPr and FDPu), abductor pollicis longus (AbPL), extensor digitorum-2,3 (ED23), extensor digitorum-4,5 (ED45), extensor digitorum communis (EDC)], wrist muscles [flexor carpi radialis (FCR), flexor carpi ulnaris (FCU), palmaris longus (PL), extensor carpi radialis longus and brevis (ECRl and ECRb), extensor carpi ulnaris (ECU)], and elbow muscles [brachioradialis (BRD), pronator teres (PT), biceps brachii (biceps), and triceps brachii (triceps)]. For monkey U, EMG wires were acutely inserted percutaneously in two muscles (FDI and AbDM) on each experimental day. The location of each EMG implant was confirmed by evoking joint and muscle movement using low intensity electrical stimulations applied through the wire electrodes during and after surgery. The recorded muscles from each monkey are tabulated in Table 1.

Data recording. During each recording session, the monkey's head was fixed to the chair with plastic rods and a glass-insulated tungsten or Elgiloy microelectrode (impedance: $1-2 \mathrm{M} \Omega$ at $1 \mathrm{kHz}$ ) was inserted into the cervical spinal cord (Fig. 1C). The position of the electrode was controlled using a hydraulic microdrive (MO-951, Narishige Scientific In- 
Table 1. Muscles recorded for each monkey

\begin{tabular}{|c|c|c|c|c|}
\hline \multirow[b]{2}{*}{ Group } & \multirow[b]{2}{*}{ Muscle } & \multicolumn{3}{|c|}{ Monkey } \\
\hline & & $A$ & $\mathrm{E}$ & U \\
\hline \multirow[t]{7}{*}{ Intrinsic hand } & FDI & $\sqrt{ }$ & $\sqrt{ }$ & $\downarrow$ \\
\hline & 2DI & & $\sqrt{ }$ & \\
\hline & 3DI & & $\sqrt{ }$ & \\
\hline & 4DI & & $\sqrt{ }$ & \\
\hline & ADP & $\sqrt{ }$ & $\sqrt{ }$ & \\
\hline & $A b P B$ & $\sqrt{ }$ & $\sqrt{ }$ & \\
\hline & AbDM & $\sqrt{ }$ & $\sqrt{ }$ & $\checkmark$ \\
\hline \multirow[t]{7}{*}{ Extrinsic hand } & FDS & $\sqrt{ }$ & $\sqrt{ }$ & \\
\hline & $\mathrm{FDPr}$ & $\sqrt{ }$ & $\sqrt{ }$ & \\
\hline & $\mathrm{FDPu}$ & $\sqrt{ }$ & $\sqrt{ }$ & \\
\hline & $A b P L$ & $\sqrt{ }^{*}$ & $\sqrt{ }$ & \\
\hline & ED23 & $\sqrt{ }$ & & \\
\hline & ED45 & $\sqrt{ }$ & $\sqrt{ }$ & \\
\hline & $\mathrm{EDC}$ & $\sqrt{ }$ & $\sqrt{ }$ & \\
\hline \multirow[t]{6}{*}{ Wrist } & $\mathrm{FCR}$ & $\sqrt{ }$ & $\sqrt{ }$ & \\
\hline & FCU & $\sqrt{ }$ & $\sqrt{ }$ & \\
\hline & $P L$ & $\sqrt{ }$ & $\sqrt{ }$ & \\
\hline & $\mathrm{ECRb}$ & $\sqrt{ }$ & & \\
\hline & ECRI & $\sqrt{ }$ & $\sqrt{ }$ & \\
\hline & ECU & $\sqrt{ }$ & $\sqrt{ }$ & \\
\hline \multirow[t]{4}{*}{ Elbow } & BRD & $\sqrt{ }$ & $\checkmark$ & \\
\hline & PT & $\sqrt{ }$ & & \\
\hline & Biceps & $\sqrt{ }$ & $\sqrt{* *}^{* *}$ & \\
\hline & Triceps & & $\sqrt{ }^{* *}$ & \\
\hline
\end{tabular}

*AbPL in monkey A was noisy, probably due to a broken electrode, so it was excluded from the data. ${ }^{* *}$ In monkey $\mathrm{E}$, biceps or triceps were arbitrarily chosen for each recording day.

strument Lab) and a custom-made X-Y stage, both of which were mounted on the recording chamber. The signal from the microelectrode was amplified $(\times 1000-10,000)$ and filtered $(300 \mathrm{~Hz}-10 \mathrm{kHz})$ using a differential amplifier (model 1800, A-M Systems) for neuron signals. A silver ball electrode was placed on the scar tissue overlying the cord surface and used as a reference electrode. Action potentials of one or more spinal neurons were isolated from background activities on-line from the neuron signal using a spike-sorting device based on a templatematching algorithm (MSD, Alpha Omega Engineering). The quality of isolation was continuously monitored throughout the recording. The stationarity of single-cell activity was checked by inspecting the average rate and the task-related firing modulation of each cell. Cells that showed an obvious trial-by-trial variance in these parameters were omitted from the study. Neuron signal and spike detection timestamps were digitized at $20 \mathrm{kHz}$. EMGs were amplified and filtered using a multichannel differential amplifier $(\times 3000-25,000,5 \mathrm{~Hz}-3 \mathrm{kHz}$; SS-6110 Nihon Kohden) and digitized at $5 \mathrm{kHz}$ concurrently with signals from potentiometers, strain gauges, touch sensors, and task events (sampling rate: $1 \mathrm{kHz}$ ). Digitized signals were stored on a hard disk for further analysis.

Spike-triggered averaging. To discriminate the PreM-INs from the neural population, a spike-triggered EMG average (STA) was computed for each neuron. Before compiling the spike-triggered averages, the spike timestamp signal was shifted off-line to correct the time lag between the action potential onset and spike detection timing (i.e., the time used in on-line spike discrimination). For some spike signals, the waveform from the neuron signal was further discriminated off-line using customwritten, cluster-cutting software to refine the spike sorting. STAs were compiled off-line for neuron-muscle pairs with at least 2,000 recorded action potentials. EMG was rectified and averaged over an $80 \mathrm{~ms}$ interval beginning $30 \mathrm{~ms}$ before and ending $50 \mathrm{~ms}$ after the spike. The baseline STA trend was subtracted using the incremented-shifted averages (ISA) method (Davidson et al., 2007b), and then the STA was smoothed with a flat five-point finite impulse response filter. Significant STA effects were identified with multiple-fragment statistical analysis (Poliakov and Schieber, 1998). The test window for this analysis was set at $12 \mathrm{~ms}$ duration (between 3 and $15 \mathrm{~ms}$ after the spinal neuron spike), because previous studies have shown that the peaks and troughs of postspike effects in spinal STAs typically occur during this time window (Perlmutter et al., 1998).

Initially, STAs were formed using all spikes recorded from a given spinal neuron. If any muscle showed a peak (or trough) significant at the $p<0.0025$ ( $p<0.05$ after Bonferroni's correction), then we accepted that neuron as producing effects. Excluding spikes that occur without EMG activity eliminates sweeps containing only noise and provides an STA that more accurately reflects the effect of the neuron on EMG activity (McKiernan et al., 1998). Therefore, STA was performed again using spikes that occurred when the target muscle EMG was larger than baseline. The root mean square (RMS) value of the target EMG was calculated using an $\sim 1$-s-long signal during intertrial intervals in which no obvious EMG was contained (RMS noise level). The RMS value of the target EMG was also calculated from $30 \mathrm{~ms}$ before to $50 \mathrm{~ms}$ after each spike, and the spike was accepted as a trigger only if the RMS value of the EMG was $>1.25$ times the RMS noise level ("EMG-filtering"). If the peak (or trough) in this EMG-filtered STA was significant at $p<0.05$ (without Bonferroni's correction), the effect was retained for further analysis.

A computer algorithm performed the following computations for each STA with a significant peak or trough. As illustrated in Figure $2 A$, the mean $\pm 2 \mathrm{SD}$ of the average was calculated from the baseline period (from 30 to $10 \mathrm{~ms}$ before the trigger). The maximum value of the peak (or minimum of a trough) within the test window was identified, and the time of the rising (falling) and falling (rising) threshold relative to the 2 SD of baseline mean was defined as the onset and offset of peak (trough). The mean percent increase (MPI) of the STA effect was calculated by averaging the STA waveform amplitude from the onset to offset, subtracting the baseline mean, and then dividing the result by the baseline mean and multiplying by 100 . Peak width at half-maximum (PWHM) of the STA effect was determined by finding the level one-half of the peak amplitude above (or below for a trough) the baseline mean and measuring the width of the peak (or trough) at this level.

We evaluated potential cross talk between simultaneously recorded EMGs by combining a cross-correlation method (Buys et al., 1986) and third differentiation of EMGs (Kilner et al., 2002). Larger motor units in a given EMG channel were isolated and used as triggers to create a triggered average of all EMG channels recorded simultaneously. The triggers were created by setting a rising threshold at just above half of the maximum amplitude. The ratio of the peak-to-peak amplitude of the averaged EMG waveform in a test channel to that in the trigger channel provided an estimate of cross talk from the trigger to the test channel. We considered that the STA effect recorded in a test channel might be attributable to cross talk from motor units recorded in the trigger channel if the ratio of the STA MPI in the test channel to that in the trigger channel was less than twice the ratio of the averaged motor unit waveform in the test channel to that in the trigger channel. When significant STA effects were identified in multiple muscles of the same recording, one of any pair of effects potentially resulting from cross talk between EMG recordings was eliminated from the present dataset. In the original study, Buys et al. (1986) used unrectified EMGs for creating triggered averages. However, the effects of motor unit synchronization could be contaminated on the triggered averages if the unrectified EMGs are used (Kilner et al., 2002). Kilner et al. (2002) showed that the third differentiation of EMGs avoids contamination of motor unit synchronization from the cross-correlations of the EMG signals (Kilner et al., 2002). Therefore, in this study, the third differentiated EMGs were used for evaluating the level of cross talk.

Intraspinal microstimulation. Intraspinal microstimuli (ISMSs) were delivered through the recording electrode in monkeys A and $\mathrm{E}$ to determine the earliest possible latency of the postspike effects from spinal PreM-INs. Single-pulse stimuli (200 $\mu$ s biphasic pulses $) \leq 30 \mu$ A often elicited responses in hand and arm muscles that were detected by stimulus-triggered averaging (Fig. $2 \mathrm{~B}$ ). The earliest facilitatory effects probably were due to the direct excitation of proximal regions of the motoneuron axons. The distribution of onset latencies of 414 poststimulus effects elicited from 56 stimulation sites in monkeys A and $\mathrm{E}$ with stimuli $\leq 30 \mu \mathrm{A}$ is shown in Figure $2 C$; the earliest poststimulus latency was $2.8 \mathrm{~ms}$. No clear difference was observed in the distributions between 


\section{A Spike-triggered average}

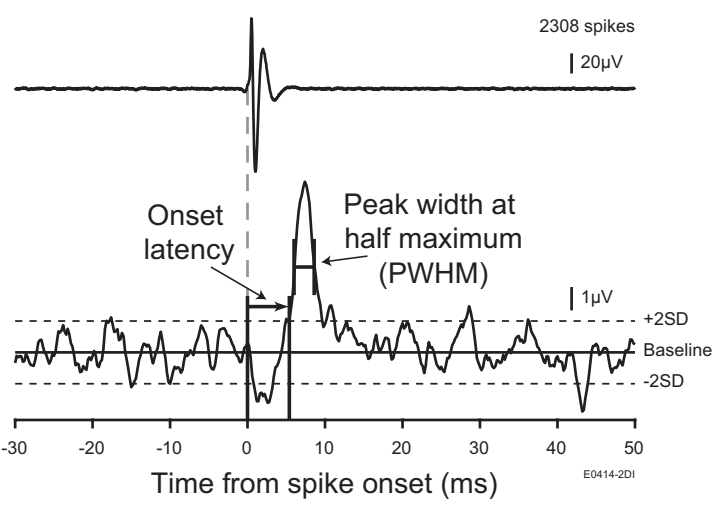

\section{B Stimulus-triggered average}
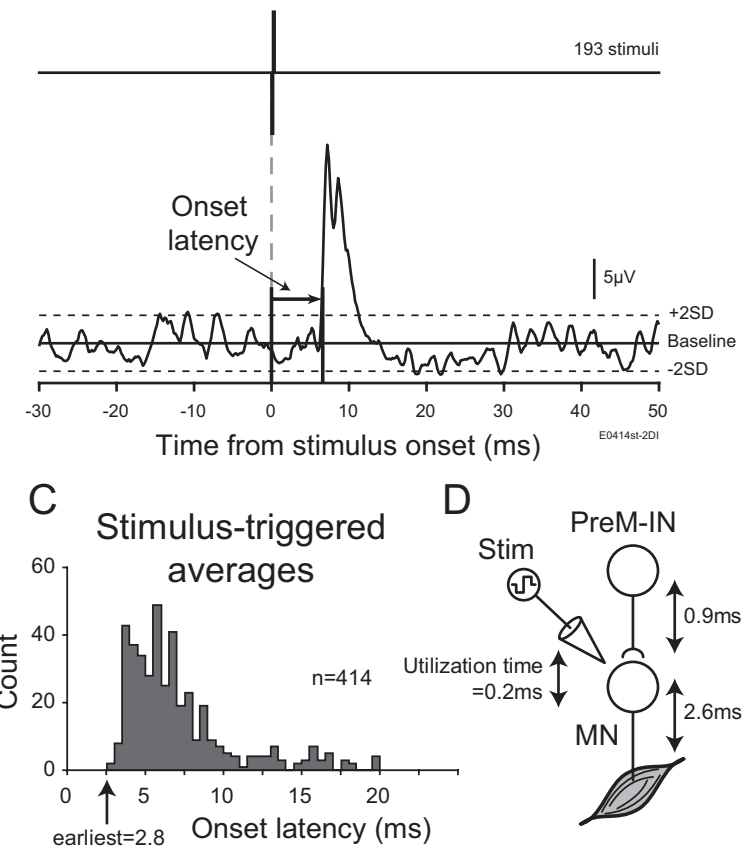

Figure 2. A, Measurement-rectified EMG spike-triggered averages. Onset was identified at the time point when the peak (or trough) crossed within \pm 2 SD of the baseline mean. Onset latency was measured relative to the onset of the triggered neuron action potential. Peak width at half-maximum was measured as the width of the peak (trough) halfway between its maximal (minimal) value and the baseline mean. $\boldsymbol{B}$, Example of rectified EMG stimulus-triggered averages. Intraspinal microstimuli delivered through the recording electrode in monkey E. Onset was identified as the time point when the peak crossed within +2 SD of the baseline mean. C, Onset latency histogram of the 414 poststimulus effects elicited from 56 stimulation sites with intensities $\leq 30 \mu \mathrm{A}$ (monkeys $\mathrm{A}$ and E). The shortest poststimulus latency was $2.8 \mathrm{~ms}$. D, Determination of the latency criterion for postspike effects of spinal PreM-INs. The utilization time $(0.2 \mathrm{~ms})$ was subtracted from the earliest poststimulus latency, and the delay between the last-order interneuron spike and spike initiation in the motoneuron (MN) $(0.9 \mathrm{~ms})$ was added. The resulting $3.5 \mathrm{~ms}$ was an estimation of the possible earliest onset of the postspike effects from spinal PreM-INs. Stim, Stimulation.

the two monkeys. The latency of these effects included the utilization time, i.e., the delay between stimulus onset and neuron discharge. We set the utilization time to 0.2 and $0.9 \mathrm{~ms}$ for the additional delay between a last-order interneuron spike and spike initiation in the motoneuron (Perlmutter et al., 1998) (Fig. 2D). Thus, we estimated that the possible earliest latency of the postspike effects from spinal PreM-INs could be $\geq 3.5 \mathrm{~ms}$.

Classification of postspike effect and synchrony effect. Two types of STA effects have been reported: postspike effects and synchrony effects (Fetz
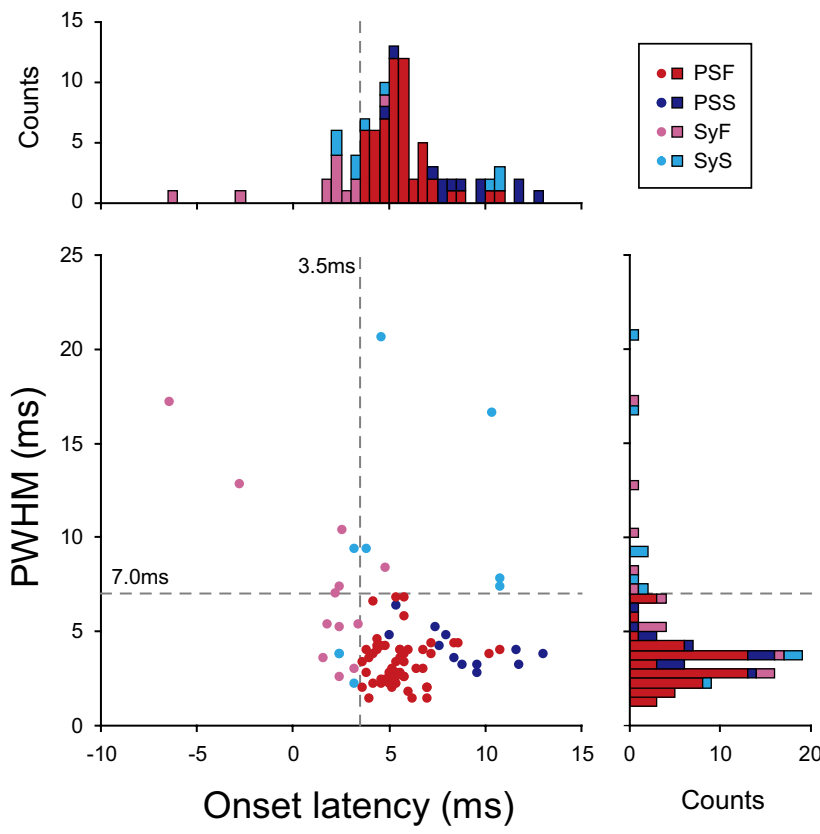

Figure 3. Scatter plot of onset latencies versus PWHM of all significant STA effects with marginal histograms $(n=89)$. Dotted crosshair is drawn at an onset latency of $3.5 \mathrm{~ms}$ and PWHM of $7 \mathrm{~ms}$, which were used to classify the postspike and synchrony effects: postspike facilitations (PSFs, red), postspike suppressions (PSSs, blue), synchrony facilitations (SyFs, magenta), and synchrony suppressions (SySs, cyan).

and Cheney, 1980; Buys et al., 1986; Mewes and Cheney, 1991; Flament et al., 1992; Perlmutter et al., 1998; Schieber and Rivlis, 2005; Davidson et al., 2007a). The postspike effect reflects the monosynaptic or disynaptic effects from the trigger neuron on the motoneuron pool that then appears as facilitation or suppression of the EMG signal (Fetz and Cheney, 1980). In contrast, the synchrony effects are derived from synaptic inputs from other neurons in the motoneuron pool that are synchronized with the discharges of the trigger neuron (Fetz and Cheney, 1980; Schieber and Rivlis, 2005). Therefore, synchrony effects can appear in STAs even if no monosynaptic or disynaptic connection exists from the trigger neuron to the motoneuron pool.

We used onset latency and the PWHM of STA effects to distinguish the postspike versus synchrony effects (Schieber and Rivlis, 2005). The earliest possible onset latency of postspike effects was set at $3.5 \mathrm{~ms}$, as described above. Based on theoretical considerations and computer simulations, a PWHM of 7-9 ms has been suggested for distinguishing postspike effects versus synchrony effects (Baker and Lemon, 1998). In this study, we used a value of $7 \mathrm{~ms}$ for the criterion of PWHM. These analyses were performed off-line using MATLAB (MathWorks).

Histological procedures. Near the end of the experiments we created a small iron deposit in the spinal cord of monkey E by passing DC $(30 \mu \mathrm{A}$ for $30 \mathrm{~s}$ ) through the Elgiloy recording electrode. At the end of each experiment, animals were killed with pentobarbital sodium $(50 \mathrm{mg} / \mathrm{kg}$ i.v.) and perfused with $10 \%$ formalin or a ferrocyanide-containing fixative $(2.25 \%$ glutaraldehyde, $0.75 \%$ formaldehyde, and $1 \%$ potassium ferrocyanide in $0.1 \mathrm{~m}$ phosphate buffer, $\mathrm{pH}$ 7.4). After fixation, the spinal cord was removed and immersed in graded sucrose solutions (final strength: $30 \%$ ). After the immersion, the spinal cord was cut into $50 \mu \mathrm{m}$ sections on a freezing microtome. To detect the iron deposit made with the Elgiloy electrodes, the sections were stained with Perl's iron reaction and then counterstained with Neutral Red to identify surrounding structures.

\section{Results}

A total of 463 spinal neurons were recorded in three monkeys (99, 342 , and 22 neurons, in monkeys $\mathrm{A}, \mathrm{E}$, and $\mathrm{U}$, respectively). Among them, 205 neurons were excluded for having $<2000$ 


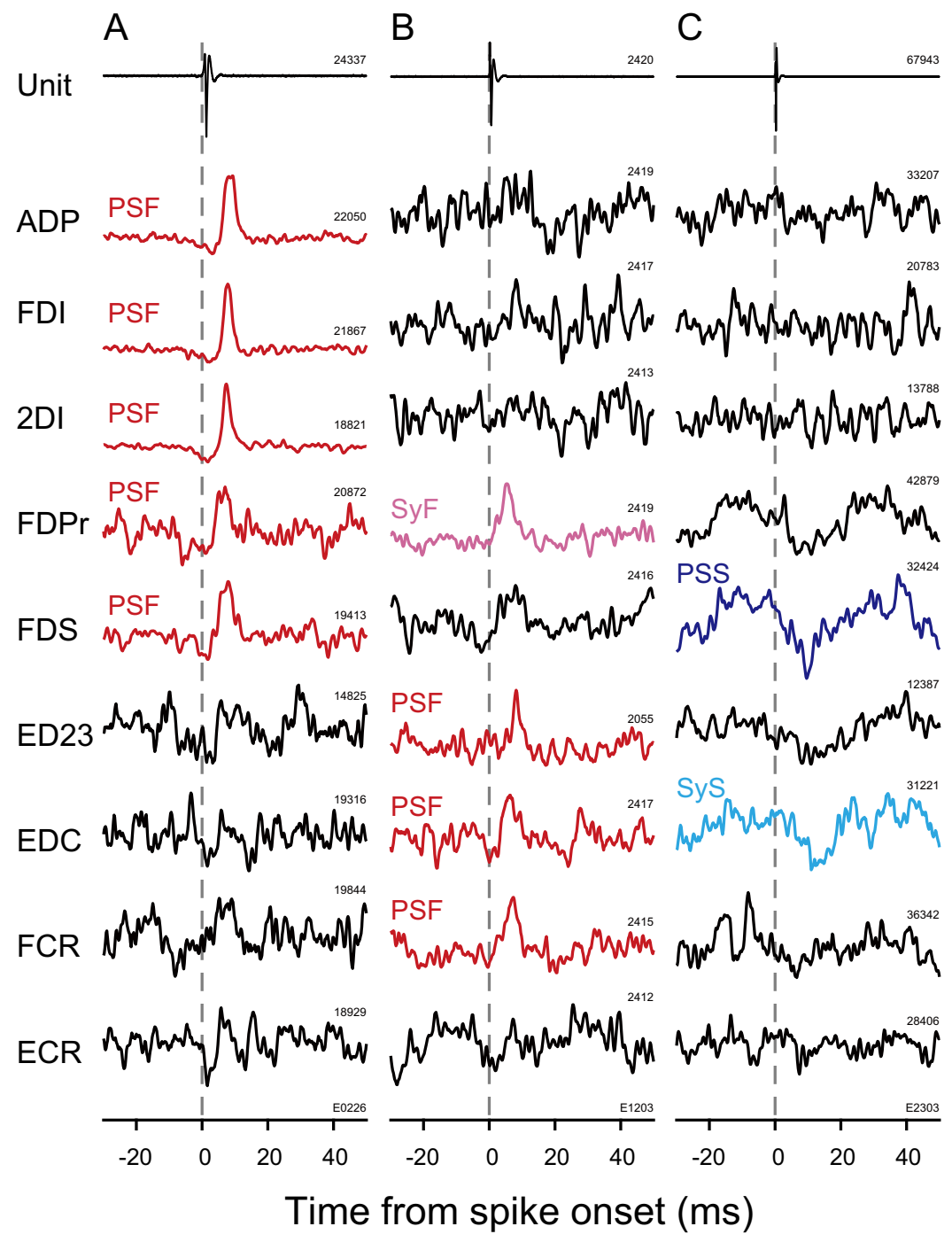

Figure 4. Spike-triggered EMG averages from hand and arm muscles. A-C, STAs in nine muscles from monkey $E$ were selected for the specimen. STAs were compiled over $80 \mathrm{~ms}(-30$ to $+50 \mathrm{~ms})$. STAs with significant PSF are shown in red, those with PSS are blue, those with SyF are magenta, those with SyS are cyan, and those with no significant effect are in black. Time 0 indicates spike onset. The numbers above each trace indicate the number of triggers used for compiling EMG-filtered STAs. All units were recorded in the $\mathrm{T} 1$ segment.

spikes. Another three neurons had a large "motor unit" signature in the STA of the unrectified EMG with only 50 spikes (Maier et al., 1998). They were identified as putative motoneurons and excluded from the dataset. As a result, 255 neurons (4821 neuron-muscle pairs) were analyzed for spike-triggered averaging.

\section{Classification of STA effects as a postspike effect or synchrony effect}

Thirty neurons $(6,22$, and 2 neurons in monkeys A, E, and $U$, respectively) produced 89 significant STA effects in hand or arm muscles. These STA effects were classified as postspike effects or synchrony effects according to their onset latency and PWHM (Fig. 3). The vast majority of STA effects $(68 / 89,76 \%)$ had a PWHM of $<7 \mathrm{~ms}$ and an onset latency of $>3.5 \mathrm{~ms}$ and were classified as postspike effects: 56 were postspike facilitations (PSFs) (Fig. 3, red) and 12 were postspike suppressions (PSSs) (Fig. 3, blue). The other effects $(21 / 89,24 \%)$ were classified as synchrony effects: 12 were synchrony facilitations (SyFs) (Fig. 3, magenta) and 9 were synchrony suppressions (SySs) (Fig. 3, cyan). Twenty-six neurons had either PSF or PSS on at least one muscle; thus, they were identified as excitatory or inhibitory premotor interneurons (PreM-IN: 21 excitatory and 5 inhibitory). No neuron had both PSF and PSS simultaneously. Four neurons had SyF or SyS, but neither PSF nor PSS, and they were identified as excitatory or inhibitory synchrony neurons (SyNs; two excitatory and two inhibitory). Again, no neurons had both SyF and SyS simultaneously.

\section{Postspike effects produced by PreM-INs}

Typical STA effects induced by three PreM-INs from monkey E are illustrated in Figure 4. The first PreM-IN (Fig. 4A), which was recorded in the $\mathrm{T} 1$ segment, produced sharp STA effects in multiple intrinsic hand muscles (ADP, FDI, and 2DI) and extrinsic hand flexors (FDPr and FDS). Their onset latencies ranged from 4.4 to $5.8 \mathrm{~ms}$ and PWHMs from 2.4 to $4.2 \mathrm{~ms}$. According to the criteria shown in Figure 3, all of these STA effects were classified as PSFs. This example clearly showed that the PreM-INs produce prominent PSFs in the finger muscles (intrinsic and extrinsic) during a precision grip task. Furthermore, the PreM-INs often produced PSFs in multiple finger muscles rather than in a single muscle. This divergent facilitation of multiple finger muscles is shown in another example (Fig. 4B). This PreM-IN (Fig. 4B) produced STA effects in multiple extrinsic hand extensors (ED23 and EDC), a flexor (FDPr), and a wrist flexor (FCR). The STA effects found in ED23, EDC, and FCR were classified as PSF, and that in the FDPr was categorized as SyF based on the onset latency and PWHM (onset latency $=1.6 \mathrm{~ms}$, PWHM $=3.6 \mathrm{~ms})$. An example of suppressive effects is shown in Figure $4 C$. This neuron produced a PSS in an extrinsic hand flexor (FDS, onset latency = $8.8 \mathrm{~ms}, \mathrm{PWHM}=3.2 \mathrm{~ms}$ ) and a SyS in an extrinsic hand extensor $(\mathrm{EDC}$, onset latency $=10.8 \mathrm{~ms}, \mathrm{PWHM}=7.4 \mathrm{~ms})$. As shown in Figure 4, PreM-INs produced prominent postspike effects in the finger muscles during a precision grip. Importantly, this is the first demonstration that spinal PreM-INs produce PSFs in intrinsic hand muscles, which is crucial for fine finger movement (Long et al., 1970; Maier and Hepp-Reymond, 1995).

To characterize these postspike effects newly found in the intrinsic hand muscles, we compared them with those of extrinsic hand, wrist, and elbow muscles (Table 2). The onset latency for intrinsic hand muscles $(6.3 \pm 1.4 \mathrm{~ms})$ was significantly longer than that for extrinsic hand $(4.7 \pm 0.9 \mathrm{~ms})$ and wrist muscles (4.6 $\pm 0.5, t$ test, $p<0.05$ with Bonferroni's correction). Similarly, peak latency for intrinsic hand muscles $(8.5 \pm 1.7 \mathrm{~ms})$ was significantly longer than that in extrinsic hand muscles $(7.1 \pm 1.6$ $\mathrm{ms})$ and wrist muscles $(6.1 \pm 1.0 \mathrm{~ms})$. These differences $(1.6-1.7$ $\mathrm{ms}$ ) between intrinsic hand muscles and extrinsic hand or wrist 
Table 2. Parameters of STA effects

\begin{tabular}{|c|c|c|c|c|c|c|c|c|c|c|c|c|c|c|c|c|c|c|c|c|}
\hline & \multicolumn{5}{|c|}{ Postspike facilitation } & \multicolumn{5}{|c|}{ Postspike suppression } & \multicolumn{5}{|c|}{ Synchrony facilitation } & \multicolumn{5}{|c|}{ Synchrony suppression } \\
\hline & $\begin{array}{l}\text { Intrinsic } \\
\text { hand }\end{array}$ & $\begin{array}{l}\text { Extrinsic } \\
\text { hand }\end{array}$ & Wrist & Elbow & Total & $\begin{array}{l}\text { Intrinsic } \\
\text { hand }\end{array}$ & $\begin{array}{l}\text { Extrinsic } \\
\text { hand }\end{array}$ & Wrist & Elbow & Total & $\begin{array}{l}\text { Intrinsic } \\
\text { hand }\end{array}$ & $\begin{array}{l}\text { Extrinsic } \\
\text { hand }\end{array}$ & Wrist & Elbow & Total & $\begin{array}{l}\text { Intrinsic } \\
\text { hand }\end{array}$ & $\begin{array}{l}\text { Extrinsic } \\
\text { hand }\end{array}$ & Wrist & Elbow & Total \\
\hline Number & 32 & 18 & 6 & - & 56 & - & 6 & 5 & 1 & 12 & 1 & 5 & 6 & - & 12 & 1 & 5 & 3 & - & 9 \\
\hline $\begin{array}{l}\text { Onset latency } \\
(\mathrm{ms})\end{array}$ & $6.3 \pm 1.4^{\dagger \ddagger}$ & $4.7 \pm 0.9^{*}$ & $4.6 \pm 0.5^{*}$ & - & $5.6 \pm 1.5$ & - & $8.7 \pm 2.2$ & $8.4 \pm 2.8$ & 11.8 & $8.8 \pm 2.4$ & 1.8 & $0.8 \pm 4.1$ & $1.9 \pm 2.5$ & - & $1.5 \pm 3.1$ & 2.4 & $7.8 \pm 3.9$ & $3.4 \pm 1.1$ & - & $5.7 \pm 3.8$ \\
\hline $\begin{array}{l}\text { Peak latency } \\
\text { (ms) }\end{array}$ & $8.5 \pm 1.7^{\text {tł }}$ & $7.1 \pm 1.6^{*}$ & $6.1 \pm 1.0^{*}$ & - & $7.8 \pm 1.8$ & - & $9.7 \pm 1.9$ & $11.2 \pm 2.0$ & 14.0 & $10.7 \pm 2.2$ & 7.6 & $6.0 \pm 1.5$ & $6.9 \pm 2.0$ & - & $6.6 \pm 1.7$ & 4.6 & $10.1 \pm 2.9$ & $6.7 \pm 2.9$ & - & $8.4 \pm 3.3$ \\
\hline PWHM (ms) & $3.2 \pm 1.0$ & $3.8 \pm 1.6$ & $2.6 \pm 1.1$ & - & $3.3 \pm 1.2$ & - & $3.7 \pm 0.8$ & $4.8 \pm 1.1$ & 3.2 & $4.1 \pm 1.0$ & 5.4 & $6.4 \pm 6.2$ & $8.5 \pm 2.7$ & - & $7.4 \pm 4.3$ & 3.8 & $10.1 \pm 3.7$ & $8.9 \pm 10.2$ & - & $9.0 \pm 6.1$ \\
\hline MPI (\%) & $9.5 \pm 4.3$ & $7.9 \pm 5.6$ & $8.0 \pm 6.2$ & - & $8.8 \pm 4.9$ & - & $-3.4 \pm 0.8$ & $-4.4 \pm 2.7$ & -1.6 & $-3.7 \pm 1.9$ & 22.7 & $6.8 \pm 3.7$ & $5.4 \pm 2.1$ & - & $7.4 \pm 5.5$ & -5.9 & $-7.2 \pm 4.4$ & $-5.9 \pm 3.5$ & - & $-6.6 \pm 3.6$ \\
\hline
\end{tabular}

Values are mean $\pm S D .{ }^{*}$, Significant difference versus intrinsic hand muscle; ${ }^{\dagger}$, significant difference versus extrinsic hand muscle; ${ }^{\ddagger}$, significant difference versus wrist muscle ( $p<0.05, t$ test with Bonfferoni's correction).

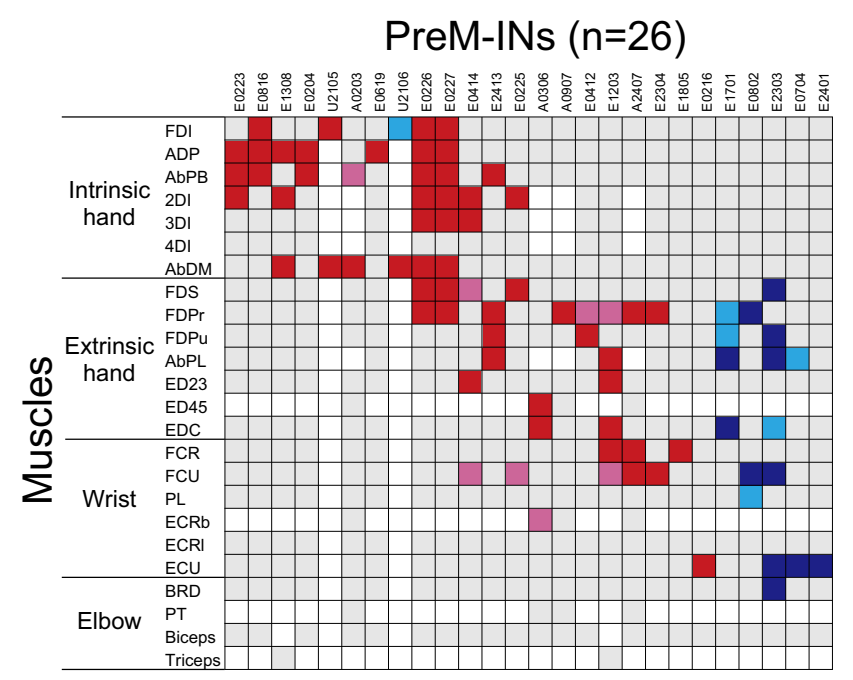

$\square$ PSF $\square$ PSS $\square$ SyF $\square$ SyS $\square$ No effect $\square$ Not recorded

Figure 5. Postspike effects of 26 PreM-INs including 21 excitatory PreM-INs and five inhibitory PreM-INs. Significant effects are color-coded: PSF, red; PSS, blue; SyF, magenta; SyS, cyan; and no significant effect, gray. The combination that was not tested is indicated as blank.

muscles could be ascribed to their anatomical locations: the muscle belly of the former is located within the hand, and those of the latter are located within the forearm. This possibility was confirmed by comparing the onset latencies of ISMS responses in each muscle group. The mean onset latencies of the earliest 30 ISMS responses in intrinsic hand, extrinsic hand, and wrist muscles were $5.6 \pm 0.7,3.9 \pm 0.3$, and $3.8 \pm 0.3 \mathrm{~ms}$, respectively (data not shown). Their difference (1.7-1.8 ms) was almost same as the difference in the PSF latencies. Therefore, the difference in PSF latencies could arise in part from differences in the peripheral conduction delay. The PSS onset and peak latencies showed no significant difference among the muscle groups, probably because both extrinsic hand and wrist muscles are located within the forearm and have similar peripheral conduction delays. Furthermore, no significant difference was found in the magnitude (MPI) or width (PWHM) among muscle groups. These results suggest that the postspike effects found in these muscle groups were similar in profile but highly different in their population, as shown below.

\section{Distribution of postspike effects in different muscle groups}

Figure 5 shows the distribution of STA effects produced by all 26 PreM-INs. Each column of the matrix indicates the STA effects produced by a given PreM-IN. The matrix exhibits several features of PreM-IN STA effects. First, single PreM-INs frequently induced postspike effects on multiple muscles rather than having an effect on a single muscle. Among the 26 Pre-INs, 17 neurons
(65\%) had PSF or PSS on more than one muscle: seven produced postspike effects in two muscles, five produced effects in three muscles, and five produced effects in four or more muscles. This divergent projection resulted in a "muscle field," which refers to the extent of the postspike effects from a single premotor neuron (Fetz and Cheney, 1980; Buys et al., 1986). Second, the postspike effects were not uniformly distributed across the recorded muscles but differently distributed according to the type of effect. PSF occurred more often in muscles primarily acting on the fingers (32/56 PSF in intrinsic hand muscles and 18/56 PSF in extrinsic hand muscles), whereas PSS mainly occurred in extrinsic hand muscles (6/12 PSS) and wrist muscles (5/12 PSS), but no PSS occurred in intrinsic hand muscles. The distribution of synchrony effects was also biased (see Table 2); almost all of the synchrony effects were observed in extrinsic hand (five SyF and five SyS) or wrist muscles (six SyF and three SyS), but few in intrinsic hand muscles (one SyF and one SyS). No synchrony effect was found in elbow muscles.

These results are summarized as an illustration in Figure $6 \mathrm{~A}$. First, PSF was most common in hand muscles. Of the 26 PreMINs, $19(73 \%)$ had PSF in intrinsic and/or extrinsic hand muscles: eight $(31 \%)$ had PSF in intrinsic hand muscles only, five (19\%) in intrinsic and extrinsic hand muscles, three $(12 \%)$ in extrinsic hand muscles only, and three (12\%) in extrinsic hand and wrist muscles. Only two $(8 \%)$ had PSFs in wrist muscles only, and no PreM-IN produced PSF in elbow muscles. In contrast, only three PreM-INs (12\%) had PSS in extrinsic hand muscles, and no PreM-IN produced PSS in intrinsic hand muscles. This result suggests that spinal PreM-INs predominantly facilitates finger muscles but rarely suppress them during a precision grip.

Figure 6, $B$ and $C$, compares the size of muscle field among the different muscle groups. The frequency of each effect (PSF or PSS) produced by a single PreM-IN was counted and averaged in each muscle group (intrinsic hand, extrinsic hand, or wrist muscle) (Fig. 6B). In general, PreM-INs had larger muscle fields in more distal hand muscles. This was confirmed by statistical analyses; the PSF muscle field was significantly larger in intrinsic hand muscles $(2.5 \pm 1.9$ muscles $)$ than in wrist muscles $(1.2 \pm 0.4$ muscles, $t$ test, $p<0.05)$. No significant difference was observed between PSF muscle fields of the intrinsic and the extrinsic hand muscles $(p=0.15)$ or in the extrinsic hand and wrist muscles $(p=0.19)$. For the PSS muscle field, no significant difference was observed between the extrinsic hand and wrist muscles $(p=$ 0.33 ). It could be argued that the comparison in Figure $6 B$ is arbitrary because the number of recorded muscles is different among the muscle groups. For example, the number of simultaneously recorded muscles was seven in the intrinsic hand muscles and five in the wrist muscles in monkey $E$. Therefore, the muscle field size was normalized by dividing the number in Figure $6 B$ by the total number of muscles recorded simultaneously in each muscle group (Fig. 6C). Even after normalization, the PSF muscle 


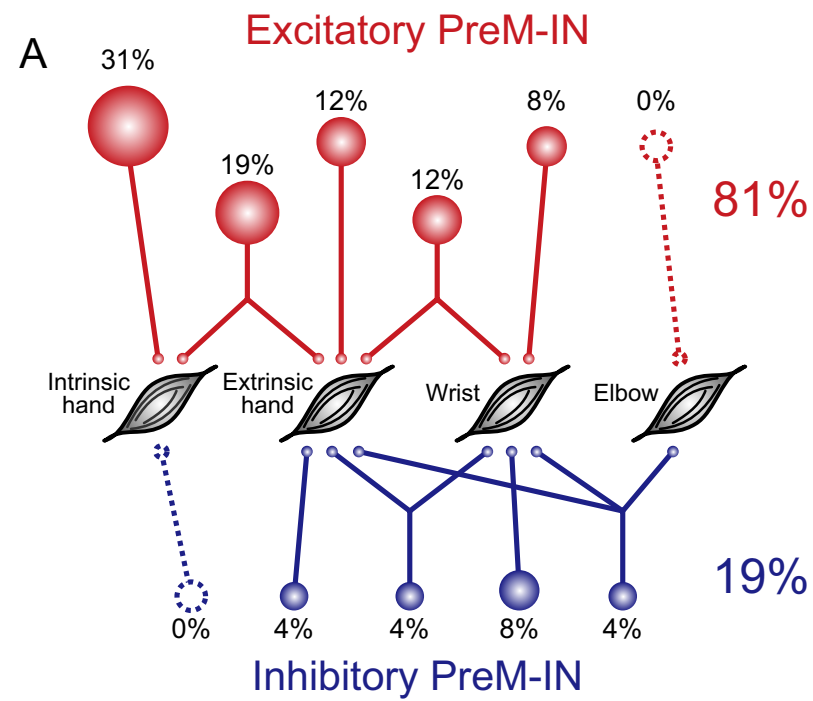

B

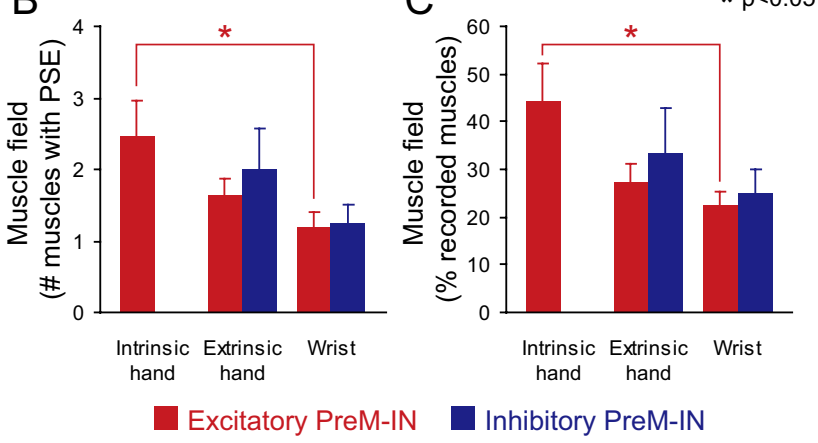

Figure 6. Distribution of postspike effects. $\boldsymbol{A}$, Relative distribution of postspike effects to intrinsic hand, extrinsic hand, wrist, and elbow muscles for 26 PreM-INs, including 21 excitatory PreM-INs (red) and five inhibitory PreM-INs (blue). Percentages of the total number of PreM-INs are indicated and depicted as relative size of associated circles. $\boldsymbol{B}$, The number of muscles simultaneously facilitated or suppressed by individual PreM-INs (muscle field size) was averaged for each muscle group. C, Muscle field size was normalized by dividing the number of muscle fields by the total number of muscles simultaneously recorded in same muscle group. Error bars indicate the SE.

field for the intrinsic hand muscles $(45 \pm 31 \%)$ was significantly larger than that for the wrist muscles $(23 \pm 6 \%)$, and no significant difference was found for the other comparisons. These results indicated that finger muscle activities during precision grip are controlled predominantly by a divergent facilitatory effect, but not by the inhibitory effects of spinal PreM-INs.

\section{Location of PreM-INs}

It is of interest whether the different types of PreM-INs exhibited any specific anatomical distribution within the spinal cord. Previous studies have stressed the technical difficulties of conventional histological reconstruction for chronic spinal recordings (Perlmutter et al., 1998; Moritz et al., 2007). Therefore, as an alternative we evaluated the intraspinal distribution of the PreMINs by comparing them with that of ISMS sites in which EMG responses were evoked in recorded muscles. Figure $7, A-C$, shows the distribution of the depth of excitatory PreM-INs (Fig. 7A), inhibitory PreM-INs (Fig. 7B), and the ISMS threshold current for evoking EMG responses (Fig. $7 C$ ). The depth was measured relative to the location where the first cellular activity was recorded for each penetration (Takei and Seki, 2008; Riddle and Baker, 2010). The result showed that the excitatory PreM-INs were distributed relatively ventral (approximate depths were 1500-3500 $\mu \mathrm{m}$ relative to the first recorded cell) (Fig. 7A), whereas the inhibitory PreM-INs were distributed relatively dorsal (0-2000 $\mu \mathrm{m}$ relative to the first recorded cell) (Fig. 7B). ISMS threshold histograms were created separately for the lower-threshold sites (Fig. 7C, $\leq 5$ $\mu \mathrm{A}$, filled bars), the intermediate-threshold sites (Fig. 7C, 6-30 $\mu \mathrm{A}$, light gray), and the higher-threshold sites (Fig. 7C, $>30 \mu \mathrm{A}$, dark gray). The lower-threshold sites were located ventrally, whereas the higher-threshold sites were localized dorsally. The intermediate-threshold sites were distributed over the entire depth. This graded ISMS threshold in the dorsoventral direction reflects that the ventrally located ISMS activates the motoneuron pool directly, whereas the dorsally located ISMS activates the motoneuron pool more indirectly. Importantly, the distribution of excitatory PreM-INs was heavily overlapped with that of the lower-threshold sites. In contrast, the inhibitory PreM-INs were more dorsally distributed and were sometimes above the higherthreshold areas. These results suggest that the excitatory PreMINs were located near the motoneuron pool, whereas the inhibitory PreM-INs were distributed in a dorsal portion distant from the motoneuron pool.

This suggestion was confirmed with an anatomical reference marked in monkey E. Figure $7 D$ shows an iron deposit made at the location where the ISMS elicited an FDPr response at a stimulus current of $>30 \mu \mathrm{A}$, but there was no response at the lower current (Fig. 7E). The deposit was made at a depth of $1490 \mu \mathrm{m}$ relative to the site where the neural activity was first recorded (Fig. 7A-C, arrowheads), and it was located around the border between the dorsal horn and the intermediate zone (Fig. 7E). As shown Figure $7, A$ and $B$, the majority of excitatory PreM-INs were located deeper than this reference point, whereas the inhibitory PreM-INs were distributed around this reference. These results further suggest that the excitatory PreM-INs were located in the intermediate zone or the ventral horn, whereas inhibitory PreM-INs were distributed over the dorsal horn and the intermediate zone.

\section{Discussion}

In this study, we examined the contribution of spinal INs in the control of grasping movements by identifying spinal premotor interneuron postspike effects (PreM-INs) in finger muscles while monkeys performed a precision grip task. We found that a significant number of PreM-INs had postspike effects in the finger muscles (Fig. 6A) and that their postspike effects in the finger muscles were mostly facilitation (postspike facilitation, PSF), but few suppressive effects (postspike suppression, PSS) were observed (Fig. 6A). Additionally, these PreM-INs often facilitated multiple finger muscles simultaneously rather than a single muscle (Figs. 4, 5, 6B, C). The number of spinal PreM-INs identified by this study was relatively small (26 PreM-INs), and the segmental location of our recordings, which was relatively focused on the lower cervical segments (C7-T1) where the motor nuclei of finger muscles are located (Jenny and Inukai, 1983), may have caused an underestimation of the postspike effects on the proximal muscles. However, recorded PreM-INs have consistently shown divergent facilitative effects on finger muscles. Therefore, we suggest that spinal INs have a significant role in generating and coordinating finger muscle activities during grasping movements.

\section{Spinal premotor neurons to finger muscles}

Premotor neurons that drive the motoneurons innervating finger muscles have been found in the motor cortex (Muir and Lemon, 
1983; Buys et al., 1986; Maier et al., 1993; McKiernan et al., 1998), red nucleus (Mewes and Cheney, 1991; Sinkjaer et al., 1995), and reticular formation (Riddle et al., 2009). According to the output effect pattern, it has been proposed that these neurons have specialized functions for the control of grasping. For example, premotor neurons in the M1, i.e., corticomotoneuronal or CM cells, have a significant role for fractionizing individual finger movements (Buys et al., 1986; Bennett and Lemon, 1996). Premotor neurons in the red nucleus, i.e., rubromotoneuronal cells, contribute to controlling the hand during target reaching (Sinkjaer et al., 1995). Here, we found finger muscle PreM-INs in the spinal cord of monkeys performing a precision grip. PreM-INs of forelimb finger muscles occur in the cat spinal cord (Hongo et al., 1989a,b; Kitazawa et al., 1993), but their function in voluntary movement control remains unknown, as these INs have almost been exclusively examined in anesthetized preparations. Therefore, as discussed below, the new findings reported in this study could add a new perspective on the neural control of grasping.

\section{Divergent facilitation of spinal PreM-INs to finger muscles}

The PreM-INs showed more divergent muscle fields in finger muscles than in wrist muscles (Fig. $6 \mathrm{~B}, \mathrm{C}$ ). It might be argued that this difference reflects a sampling bias: during grasping, the finger muscles are more active as prime movers than are the forearm muscles, resulting in an underestimation of the forearm muscle field. However, our observations of muscle field in forearm muscles were consistent with a previous study (Perlmutter et al., 1998) that examined the postspike effects of spinal PreM-INs during a wrist flexionextension task in which forearm muscles are the prime movers. The results showed that spinal PreM-INs produced postspike effects in $\sim 30 \%$ of simultaneously recorded, coactive forearm muscles (34\% for PSF, $28 \%$ for PSS). In the present study, the forearm muscle field size was almost similar to the results of the previous study (extrinsic hand muscles: 29\% for PSF and 33\% for PSS; wrist muscles: $23 \%$ for PSF and $25 \%$ for PSS), but the muscle field size of the intrinsic hand muscles was significantly larger than these muscles ( $45 \%$ for PSF). Therefore, these comparisons allowed us to conclude that muscle field size is unrelated to whether a given muscle is a prime mover of an ongoing movement and that the divergent facilitation of spinal PreM-IN is not derived from sampling bias but is a characteristic feature of intrinsic hand muscles.

\section{Predominant facilitations by spinal PreM-INs to finger muscles}

Postspike effects produced by spinal PreM-INs in finger muscles were mainly facilitative rather than suppressive (Fig. 6A). Sixteen of the 26 (62\%) PreM-INs tested here produced postspike facili- tations on the intrinsic and/or extrinsic hand muscles (Fig. 6A). In contrast, only three (12\%) PreM-INs showed postspike suppressions on extrinsic hand muscles, and no PreM-INs showed PSS in intrinsic hand muscles (Fig. 6A). This result suggests that the effects of PreM-INs on finger muscles were biased toward facilitatory effects rather than showing a balance of facilitatory and suppressive effects. A number of factors could cause this facilitatory bias. First, postspike suppression may be underestimated if it is evaluated using the STA of EMGs (Perlmutter et al., 1998), because to identify PSSs neurons should be coactive with their target muscles even though the neurons suppress the muscles. However, this is a general limitation of the STA method, and it does not explain why the PSS was much rarer in hand muscles than in wrist muscles (Fig. 6A). Second, the anatomical difference between the connections of excitatory and inhibitory INs to the hand motoneurons may account for the facilitatory bias. In cats, a lack of recurrent collateral arbors was reported in the motoneurons innervating digital muscles of the forelimb (Hahne et al., 1988; Hörner et al., 1991) and hindlimb (Cullheim and Kellerth, 1978; McCurdy and Hamm, 1992). These observations suggest that there are fewer recurrent inhibitory neurons for distal muscles. However, no further evidence is available demonstrating that there are fewer inhibitory PreM-INs for distal muscles. 
Third, a plausible explanation for the facilitatory bias is that a greater number of excitatory and a smaller number of inhibitory PreM-INs for finger motoneurons were recruited during the precision grip task. As discussed above, excitatory PreM-INs have a divergent excitatory output to finger muscles compared with that to wrist muscles. This difference may help shape a broad coactivation of hand muscles, which is characteristic during a grasping movement, including a precision grip (Long et al., 1970; Smith, 1981; Maier and Hepp-Reymond, 1995). Recruiting PreM-INs that produce divergent facilitation of multiple finger muscles could be relevant for producing these coactive EMG patterns. This EMG coactivation could be enhanced by suppressing inhibitory PreM-INs. The primary function of inhibitory interneurons is to focus or sharpen muscle activation to achieve stricter muscle activation. For example, Ia inhibitory interneurons function to reciprocally suppress antagonist muscles concurrently with agonist activations. Therefore, it can be speculated that the inhibitory PreM-INs in finger muscles were silent or suppressed during the precision grip task to enhance coactivation of various intrinsic and extrinsic hand muscles.

\section{Building hand synergies by spinal interneuron: a hypothesis} Synergy for grasping has been proposed in human (Santello et al., 1998; Mason et al., 2001; Zatsiorsky et al., 2003; Shim et al., 2005a,b; Theverapperuma et al., 2006; Zhang et al., 2008) and monkey studies (Brochier et al., 2004; Mason et al., 2004; Overduin et al., 2008). However, the neural correlate of hand synergy is still unclear. Involvement of CM cells in the control of primate grasping has been extensively studied (Muir and Lemon, 1983; Buys et al., 1986; Lemon et al., 1986; Bennett and Lemon, 1996; Lemon, 2008), and it is now widely accepted that CM cells crucially contribute to fractionating the finger muscles for relatively independent finger movements. Because CM cells have muscle fields in small but specific groups of synergistic finger muscles (Buys et al., 1986), it is possible that CM cells could function to coordinate synergistic muscle activities. Spinal PreM-INs could be another candidate for the premotor forces that create hand synergies for grasping. The present study showed that a significant number of spinal PreM-INs have PSF on finger muscles and that the muscle fields are characteristically larger in intrinsic hand muscles. These divergent facilitations from PreM-INs could help form "functional units" for hand movements.

Another line of evidence has accumulated for the relationship of spinal interneurons to synergies. Bizzi et al. proposed that the synergy for leg movement of frogs is formed in the spinal interneurons (Bizzi et al., 1991, 2002; Giszter et al., 1993; Mussa-Ivaldi et al., 1994; Tresch et al., 1999; Saltiel et al., 2001). Recently, Hart and Giszter (2010) showed that divergent projections of spinal PreM-INs in the frog lumbar region are well correlated with muscle synergy in both spatial projection and temporal activity (Hart and Giszter, 2010). These results suggest that spinal interneurons may be contributing to reduce the high degree of freedom that is intrinsic to the primate hand by shaping muscle synergy with divergent facilitatory projections. This hypothesis will be tested in further studies investigating the relationship between spinal PreM-INs activities and muscle synergies during grasping movements.

\section{Conclusions}

We have identified spinal PreM-IN populations that had postspike effects on intrinsic hand muscles in monkeys performing a precision grip task. These PreM-INs often produced divergent facilitation in multiple intrinsic hand muscles. The results suggest that spinal interneurons function to coactivate multiple finger muscles, forming synergies to control grasping.

\section{References}

Armand J, Olivier E, Edgley SA, Lemon RN (1997) Postnatal development of corticospinal projections from motor cortex to the cervical enlargement in the macaque monkey. J Neurosci 17:251-266.

Baker SN, Lemon RN (1998) Computer simulation of post-spike facilitation in spike-triggered averages of rectified EMG. J Neurophysiol 80:1391-1406.

Baldissera F, Hultborn H, Illert M (1981) Integration in spinal neuronal systems. In: Handbook of physiology, Sec I, The nervous system, Vol II, Motor control, Part I (Brooks VB, ed), pp 509-595. Bethesda, MD: American Physiological Society.

Bell C (1834) The hand, its mechanism and vital endowments, as evincing design. London: William Pickering.

Bennett KM, Lemon RN (1996) Corticomotoneuronal contribution to the fractionation of muscle activity during precision grip in the monkey. J Neurophysiol 75:1826-1842.

Bizzi E, Mussa-Ivaldi FA, Giszter S (1991) Computations underlying the execution of movement: a biological perspective. Science 253:287-291.

Bizzi E, D’Avella A, Saltiel P, Tresch M (2002) Modular organization of spinal motor systems. Neuroscientist 8:437-442.

Bortoff GA, Strick PL (1993) Corticospinal terminations in two new-world primates: further evidence that corticomotoneuronal connections provide part of the neural substrate for manual dexterity. J Neurosci 13:5105-5118.

Brochier T, Spinks RL, Umilta MA, Lemon RN (2004) Patterns of muscle activity underlying object-specific grasp by the macaque monkey. J Neurophysiol 92:1770-1782.

Buys EJ, Lemon R, Mantel GW, Muir RB (1986) Selective facilitation of different hand muscles by single corticospinal neurones in the conscious monkey. J Physiol 381:529-549.

Cheema SS, Rustioni A, Whitsel BL (1984) Light and electron microscopic evidence for a direct corticospinal projection to superficial laminae of the dorsal horn in cats and monkeys. J Comp Neurol 225:276-290.

Cullheim S, Kellerth JO (1978) A morphological study of the axons and recurrent axon collaterals of cat alpha-motoneurones supplying different hind-limb muscles. J Physiol 281:285-299.

Davidson AG, Schieber MH, Buford JA (2007a) Bilateral spike-triggered average effects in arm and shoulder muscles from the monkey pontomedullary reticular formation. J Neurosci 27:8053-8058.

Davidson AG, O’Dell R, Chan V, Schieber MH (2007b) Comparing effects in spike-triggered averages of rectified EMG across different behaviors. J Neurosci Methods 163:283-294.

Dum RP, Strick PL (1996) Spinal cord terminations of the medial wall motor areas in macaque monkeys. J Neurosci 16:6513-6525.

Fetz EE, Cheney PD (1980) Postspike facilitation of forelimb muscle activity by primate corticomotoneuronal cells. J Neurophysiol 44:751-772.

Flament D, Fortier PA, Fetz EE (1992) Response patterns and postspike effects of peripheral afferents in dorsal root ganglia of behaving monkeys. J Neurophysiol 67:875-889.

Giszter SF, Mussa-Ivaldi FA, Bizzi E (1993) Convergent force fields organized in the frog's spinal cord. J Neurosci 13:467-491.

Hahne M, Illert M, Wietelmann D (1988) Recurrent inhibition in the cat distal forelimb. Brain Res 456:188-192.

Hart CB, Giszter SF (2010) A neural basis for motor primitives in the spinal cord. J Neurosci 30:1322-1336.

Hongo T, Kitazawa S, Ohki Y, Xi MC (1989a) Functional identification of last-order interneurones of skin reflex pathways in the cat forelimb segments. Brain Res 505:167-170.

Hongo T, Kitazawa S, Ohki Y, Sasaki M, Xi MC (1989b) A physiological and morphological study of premotor interneurones in the cutaneous reflex pathways in cats. Brain Res 505:163-166.

Hörner M, Illert M, Kümmel H (1991) Absence of recurrent axon collaterals in motoneurones to the extrinsic digit extensor muscles of the cat forelimb. Neurosci Lett 122:183-186.

Jankowska E (1992) Interneuronal relay in spinal pathways from proprioceptors. Prog Neurobiol 38:335-378.

Jenny AB, Inukai J (1983) Principles of motor organization of the monkey cervical spinal cord. J Neurosci 3:567-575.

Kilner JM, Baker SN, Lemon RN (2002) A novel algorithm to remove elec- 
trical cross-talk between surface EMG recordings and its application to the measurement of short-term synchronisation in humans. J Physiol 538:919-930.

Kitazawa S, Ohki Y, Sasaki M, Xi M, Hongo T (1993) Candidate premotor neurones of skin reflex pathways to $\mathrm{T} 1$ forelimb motoneurones of the cat. Exp Brain Res 95:291-307.

Kuypers H (1981) Anatomy of the descending pathways. In: Handbook of physiology, Sec I, The nervous system, Vol II, Motor control, Part I (Brooks V, ed), pp 597-666. Bethesda: American Physiological Society.

Kuypers HG, Brinkman J (1970) Precentral projections to different parts of the spinal intermediate zone in the rhesus monkey. Brain Res 24:29-48.

Lawrence DG, Kuypers HG (1968) The functional organization of the motor system in the monkey. I. The effects of bilateral pyramidal lesions. Brain 91:1-14.

Lemon RN (2008) Descending pathways in motor control. Annu Rev Neurosci 31:195-218.

Lemon RN, Mantel GW, Muir RB (1986) Corticospinal facilitation of hand muscles during voluntary movement in the conscious monkey. J Physiol 381:497-527.

Long C 2nd, Conrad PW, Hall EA, Furler SL (1970) Intrinsic-extrinsic muscle control of the hand in power grip and precision handling: an electromyographic study. J Bone Joint Surg Am 52:853-867.

Maier MA, Hepp-Reymond MC (1995) EMG activation patterns during force production in precision grip. I. Contribution of 15 finger muscles to isometric force. Exp Brain Res 103:108-122.

Maier MA, Bennett KM, Hepp-Reymond MC, Lemon RN (1993) Contribution of the monkey corticomotoneuronal system to the control of force in precision grip. J Neurophysiol 69:772-785.

Maier MA, Perlmutter SI, Fetz EE (1998) Response patterns and force relations of monkey spinal interneurons during active wrist movement. J Neurophysiol 80:2495-2513.

Mason CR, Gomez JE, Ebner TJ (2001) Hand synergies during reach-tograsp. J Neurophysiol 86:2896-2910.

Mason CR, Theverapperuma LS, Hendrix CM, Ebner TJ (2004) Monkey hand postural synergies during reach-to-grasp in the absence of vision of the hand and object. J Neurophysiol 91:2826-2837.

McCurdy ML, Hamm TM (1992) Recurrent collaterals of motoneurons projecting to distal muscles in the cat hindlimb. J Neurophysiol 67: 1359-1366.

McKiernan BJ, Marcario JK, Karrer JH, Cheney PD (1998) Corticomotoneuronal postspike effects in shoulder, elbow, wrist, digit, and intrinsic hand muscles during a reach and prehension task. J Neurophysiol 80:1961-1980.

Mewes K, Cheney PD (1991) Facilitation and suppression of wrist and digit muscles from single rubromotoneuronal cells in the awake monkey. J Neurophysiol 66:1965-1977.

Moritz CT, Lucas TH, Perlmutter SI, Fetz EE (2007) Forelimb movements and muscle responses evoked by microstimulation of cervical spinal cord in sedated monkeys. J Neurophysiol 97:110-120.

Muir RB, Lemon RN (1983) Corticospinal neurons with a special role in precision grip. Brain Res 261:312-316.

Mussa-Ivaldi FA, Giszter SF, Bizzi E (1994) Linear combinations of primitives in vertebrate motor control. Proc Natl Acad Sci U S A 91:7534-7538.

Napier JR (1956) The prehensile movements of the human hand. J Bone Joint Surg Br 38:902-913.
Overduin SA, d'Avella A, Roh J, Bizzi E (2008) Modulation of muscle synergy recruitment in primate grasping. J Neurosci 28:880-892.

Perlmutter SI, Maier MA, Fetz EE (1998) Activity of spinal interneurons and their effects on forearm muscles during voluntary wrist movements in the monkey. J Neurophysiol 80:2475-2494.

Poliakov AV, Schieber MH (1998) Multiple fragment statistical analysis of post-spike effects in spike-triggered averages of rectified EMG. J Neurosci Methods 79:143-150.

Porter R, Lemon R (1993) Corticospinal function and voluntary movement. Oxford: Oxford UP.

Ralston DD, Ralston HJ 3rd (1985) The terminations of corticospinal tract axons in the macaque monkey. J Comp Neurol 242:325-337.

Rathelot JA, Strick PL (2009) Subdivisions of primary motor cortex based on cortico-motoneuronal cells. Proc Natl Acad Sci U S A 106:918-923.

Riddle CN, Baker SN (2010) Convergence of pyramidal and medial brain stem descending pathways onto macaque cervical spinal interneurons. J Neurophysiol 103:2821-2832.

Riddle CN, Edgley SA, Baker SN (2009) Direct and indirect connections with upper limb motoneurons from the primate reticulospinal tract. J Neurosci 29:4993-4999.

Robinson FR, Houk JC, Gibson AR (1987) Limb specific connections of the cat magnocellular red nucleus. J Comp Neurol 257:553-577.

Saltiel P, Wyler-Duda K, D’Avella A, Tresch MC, Bizzi E (2001) Muscle synergies encoded within the spinal cord: evidence from focal intraspinal NMDA iontophoresis in the frog. J Neurophysiol 85:605-619.

Santello M, Flanders M, Soechting JF (1998) Postural hand synergies for tool use. J Neurosci 18:10105-10115.

Schieber MH, Rivlis G (2005) A spectrum from pure post-spike effects to synchrony effects in spike-triggered averages of electromyographic activity during skilled finger movements. J Neurophysiol 94:3325-3341.

Shim JK, Latash ML, Zatsiorsky VM (2005a) Prehension synergies in three dimensions. J Neurophysiol 93:766-776.

Shim JK, Latash ML, Zatsiorsky VM (2005b) Prehension synergies: trial-totrial variability and principle of superposition during static prehension in three dimensions. J Neurophysiol 93:3649-3658.

Sinkjaer T, Miller L, Andersen T, Houk JC (1995) Synaptic linkages between red nucleus cells and limb muscles during a multi-joint motor task. Exp Brain Res 102:546-550.

Smith AM (1981) The coactivation of antagonist muscles. Can J Physiol Pharmacol 59:733-747.

Takei T, Seki K (2008) Spinomuscular coherence in monkeys performing a precision grip task. J Neurophysiol 99:2012-2020.

Takei T, Seki K (2009) Post-spike effects of spinal interneurons on hand muscles in monkeys performing a precision grip. Soc Neurosci Abstr 35:10.19.

Theverapperuma LS, Hendrix CM, Mason CR, Ebner TJ (2006) Finger movements during reach-to-grasp in the monkey: amplitude scaling of a temporal synergy. Exp Brain Res 169:433-448.

Tresch MC, Saltiel P, Bizzi E (1999) The construction of movement by the spinal cord. Nat Neurosci 2:162-167.

Zatsiorsky VM, Gao F, Latash ML (2003) Prehension synergies: effects of object geometry and prescribed torques. Exp Brain Res 148:77-87.

Zhang W, Scholz JP, Zatsiorsky VM, Latash ML (2008) What do synergies do? Effects of secondary constraints on multidigit synergies in accurate force-production tasks. J Neurophysiol 99:500-513. 